\title{
Revolution in UK General Practice Due to COVID- 19 Pandemic: A Cross-Sectional Survey
}

Sanjeev C. Sharma ${ }^{1}$, Sonita Sharma ${ }^{2}$, Arjuna Thakker ${ }^{3}$, Gopal Sharma ${ }^{4}$, Mohamed Roshan ${ }^{5}$, Vivek Varakantam $^{6}$

1. Family Medicine, Whittington Health NHS Trust, London, GBR 2. Family Medicine, University of Buckingham Medical School, Buckingham, GBR 3. Family Medicine, University Hospitals of Leicester NHS Trust, Leicester, GBR 4. Family Medicine, Fosse Medical Centre, Leicester, GBR 5. Family Medicine, Willows Health, Leicester, GBR 6. Family Medicine, Croft Medical Centre, Leicester, GBR

Corresponding author: Sanjeev C. Sharma, scsharma1@me.com

\section{Abstract}

\section{Objectives}

To assess how UK General Practitioners (GPs) and Practice Managers (PMs) have coped with the challenges posed by the coronavirus disease-19 (COVID-19) pandemic and whether they felt adequately supported by the wider National Health Service (NHS).

\section{Methods}

This is a cross-sectional survey. All GPs and PMs (total 1,354) in Leicester, Leicestershire, and Rutland (LLR) were invited to participate in an online questionnaire.

\section{Results}

A total of 95 invitees completed the survey. Over a quarter had required time off work due to COVID symptoms or contact. All respondents described either introducing or increasing the use of remote patient consultations. Most striking was the rise in video consultations from just $3 \%$ to $95 \%$ during the pandemic. Almost half of the feedback on the usefulness of remote consultations were positive, $16 \%$ were negative and $17 \%$ were mixed. The most commonly cited benefit was time efficiency. Drawbacks of remote consultations included technical difficulties and poor patient communication. Practice premises, systems and processes also required significant modifications during the pandemic to ensure the provision of safe clinical care, including reception screens, one-way patient flow, greater infection prevention measures. However, despite their ability to introduce such widescale change virtually overnight, over $10 \%$ of respondents reported that the strain had placed their practice at risk of closure. Over half of respondents felt they were not provided with adequate personal protective equipment (PPE) for the safety of their staff. Perception of the support provided by NHS England and the Clinical Commissioning Groups (CCGs) was rather mixed, although additional guidelines were broadly welcomed. The most requested enduring changes related to remote patient consultations (59\%) and remote triage (19\%). However, in order to support such largescale permanent change, study respondents felt that a different funding and financial structure is required together with improved IT infrastructure, greater patient education and a more supportive regulatory environment.

\section{Conclusions}

COVID-19 has substantially accelerated the pace of change within NHS primary care. The long-term fear is that there may be insufficient financial and clinical backing from regulatory bodies to support such rapid and far-reaching changes.

Categories: Family/General Practice

Keywords: covid-19, corona virus, general practice, survey research, coronavirus pandemic, primary care

\section{Introduction}

The coronavirus disease-19 (COVID-19) pandemic prompted an unprecedented restructuring and rapid adaptation of all health and social care services [1]. Delivery of primary care services is complex and has been gradually evolving since the introduction of the National Health Service (NHS) Five Year Forward View in 2014 [2] and primary care networks (PCNs) in 2019 [1,3]. However, such evolutionary change was triggered into an overnight revolution by the recent pandemic. Numerous changes were introduced such as increased usage of telemedicine [4] and closer working with NHS England and Public Health England (PHE), e.g. for personal protective equipment (PPE) guidance [5].

This cross-sectional survey aims to assess how General Practitioners (GPs) and Practice Managers (PMs) 
have coped with the challenges posed by the COVID-19 pandemic and whether they felt adequately supported by the wider NHS.

\section{Materials And Methods \\ Participants and procedures}

All GPs and PMs in Leicester, Leicestershire, and Rutland (LLR) were invited to participate in an online cross-sectional survey (supplementary figures in the Appendices) designed using Google Forms. Emails were sent to a potential 1,354 participants (1,111 GPs, $243 \mathrm{PMs})$. Responses were kept anonymous to facilitate honest feedback. Data was collected between May 26, 2020 to June 14, 2020. This was a voluntary survey of healthcare professionals, so no ethical approval was required.

\section{Online survey}

The 28-question survey included a range of open and closed questions and was developed collaboratively by the authors SCS and SMS, whilst GKS, MR, and VV evaluated the questionnaire and provided amendments. The responses were either checkbox, Likert scale (0 to 10$)$ or long answer.

Questions were designed to cover a range of domains namely: 1. Respondent demographic information, 2. Changes to consultation methods, 3. Modifications to premises, 4. Modifications to practice systems and processes, 5. Usefulness of support from governing bodies (NHS England, PHE, and Clinical Commissioning Groups (CCG)), 6. Benefits of implemented changes.

The survey was tested by a GP and a PM to establish face and content validity, who also provided feedback on ease of completion (estimated completion time 5-10 minutes). The final version was confirmed by all authors before wider dissemination.

\section{Data analysis}

Quantitative statistical analysis was performed using IBM Statistical Packing for Social Sciences (SPSS) software, version 25.0 (SPSS, Chicago, IL, USA). The qualitative data collected from the five open-ended survey questions were analysed by SCS and SMS independently to reduce bias. They initially identified common themes and then subsequently coded the responses independently. The researchers convened to discuss discrepancies. Frequencies of common themes were then recorded.

\section{Results}

\section{Respondent demographic information}

Ninety-five out of 1,354 invitees completed the online survey ( $7 \%$ response rate). All respondents reported to be working during the COVID-19 pandemic. 10\% were working completely from home while just over a third did no remote working. A significant proportion of the respondents were GP partners (46.3\%) and over $86 \%$ had more than 10 years of NHS experience. Table 1 details the full description of respondent demographic information. Over a quarter (28.4\%) had required time off work due to COVID symptoms or contact. 


\section{Cureus}

Demographic Information

Job Title

GP Partner

Salaried GP

Locum GP

Practice Manager

Number of Years NHS Experience

$<5$ years

$5-10$ years

$>10$ years

$82(86.3 \%)$

Black, Asian and Ethnic Minority (BAME) Group Member

Yes

No

$43(45.3 \%)$

Practicing in the City vs County

City

County
Number of Respondents (\%)

$44(46.3 \%)$

$14(14.7 \%)$

$5(5.3 \%)$

$32(33.7 \%)$

TABLE 1: Respondent demographic information

\section{Changes in patient consultation methods}

All respondents described either introducing or increasing the use of remote patient consultations. 81 of the 95 participants reported an increase in teleconsultations of $80-100 \%$ compared to pre-COVID levels. Figure 1 depicts the reported usage of different modes of patient consultation before, during and anticipated after the pandemic. Most striking was the rise in video consultations from just 3\% to $95 \%$ during the pandemic and almost all respondents were also planning to continue using this technology (91\%). In addition, there were significant increases in email and telephone consultations.

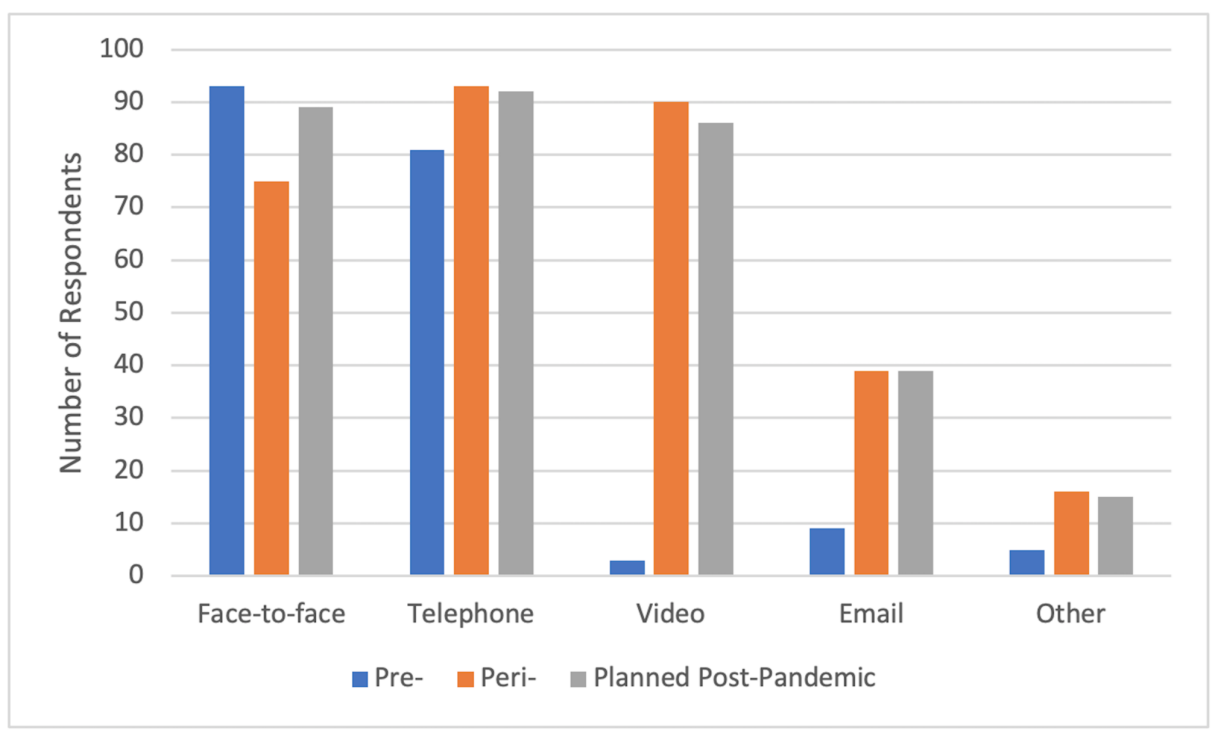


FIGURE 1: Forms of patient consultation used pre-, peri- and planned post-pandemic

As illustrated in Figure 2, satisfaction with the support provided on the use of remote technology was rather mixed but overall acceptable given that this was an unprecedented emergency requiring urgent action at all tiers of the NHS. 23 out of the 95 respondents described the support as insufficient $(<5 / 10)$ while over $75 \%$ were content with the guidance provided $(\geqslant 5 / 10)$.

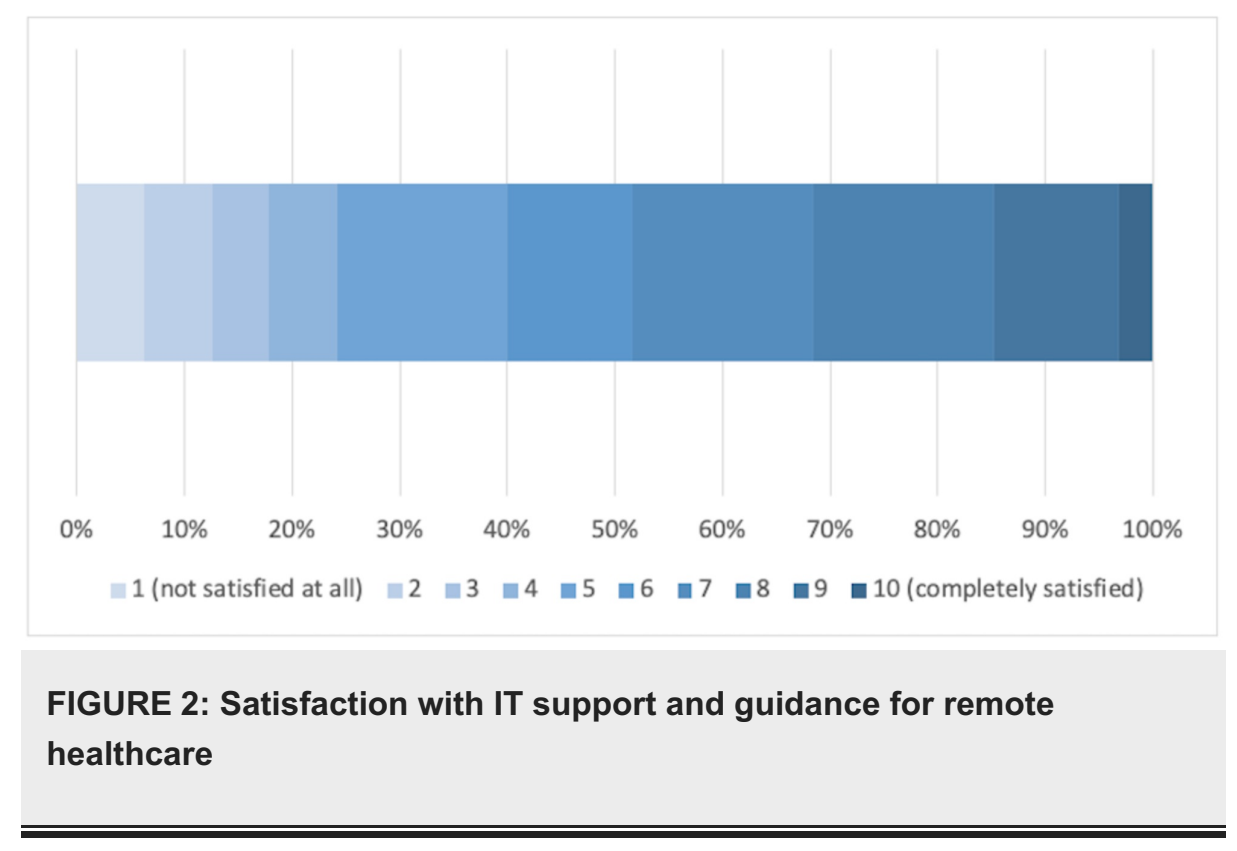

Almost half of the GP/PM feedback on the utility of remote patient consultations were positive, $16 \%$ were negative and $17 \%$ were mixed, whilst $18 \%$ failed to respond. The most commonly cited benefit was time efficiency (17\%). 13\% reported particular value in certain circumstances, such as monitoring care home patients and certifying deaths and $7 \%$ described the benefits of remote triage. Other obvious suggestions included that remote encounters were safer than face-to-face consultations (3\%) and also more flexible/convenient (3\%).

Conversely, the drawbacks of remote consultations included technical difficulties (10\%) and poor patient communication (7\%). GPs cited problems detecting non-verbal cues and language barrier issues. $6 \%$ described remote consultations as actually being time inefficient, including doubling the workload when there was need to progress to a face-to-face encounter. One doctor explained how patients were less considerate towards his/her time pressures in the absence of a busy physical waiting room; $3 \%$ felt that the video and photo technology for visualising skin lesions was of poor quality, although one GP reported the converse; $3 \%$ felt that remote consultations were potentially less safe than actually seeing a patient and one clinician highlighted the need to provide enhanced safety netting advice. Two respondents also reported concern over burn out as they found teleconsultations consultations more tiring than face-to-face meetings.

Respondents were also asked to provide any patient feedback they may have received on their increased usage of remote consultations; $56 \%$ described positive responses, $17 \%$ reported mixed feedback, whilst only $2 \%$ had received negative patient comments. Patients had highlighted various advantages such as improved access and reduced waiting time (14\%), increased convenience of consulting from home (13\%), and time efficiency (5\%). The main drawbacks for patients were technical difficulties (10\%), particularly for elderly populations, and $4 \%$ found teleconsultations impersonal compared to face-to-face meetings with their doctor. However, only $8 \%$ of respondents stated that their patients had expressed an actual preference for face-to-face consultations.

\section{Changes to practice premises}

The study revealed that practices had to make significant changes to their premises in order to continue the provision of safe services during the COVID-19 pandemic. The most commonly reported changes were reducing the number of waiting room chairs to maintain social distancing, additional cleaning, introducing a reception screen, creating separate entrance and exit doors. See Table 2 for the full breakdown on premise modifications. 


\section{Cureus}

Modifications to Premises

Reception screen

Separate entrance and exit doors

Fewer waiting room chairs

Automated doors

Automated hand driers

Additional cleaning

Increased signage

Hot and cold zones

Other (video-controlled entry, security showing patients to don and doff, reduced clutter, one-way system)
Number of Respondents

(\%)

$74(77.9 \%)$

$53(55.8 \%)$

$82(86.3 \%)$

$19(20 \%)$

$14(14.7 \%)$

$75(78.9 \%)$

$4(4.2 \%)$

$7(7.4 \%)$

$5(5.3 \%)$

\section{TABLE 2: Modifications to premises}

\section{Changes to practice systems and processes}

Table 3 shows that significant changes were also made to practice systems and processes, such as introducing staff risk stratification policies.

Changes to Systems/Processes

Longer consultation times

Cleaning gaps between patients

Staggered surgeries to avoid overcrowding

Patient masks

Hand sanitisers at entrance

Revised practice website/patient information leaflets

Greater use of patient text messaging

Staff risk stratification

Greater offsite working

Additional staff training

Revised policies and procedures

Greater administrative burden (e.g. daily reporting)

More frequent team meetings

Remote team meetings

Additional financial burden

Closer liaison with neighbouring practices

\section{Number of Respondents (\%)}

$47(49.5 \%)$

$68(71.6 \%)$

$60(63.2 \%)$

$55(57.9 \%)$

$72(75.8 \%)$

$80(84.2 \%)$

$84(88.4 \%)$

$86(90.5 \%)$

$80(84.2 \%)$

$64(67.4 \%)$

$86(90.5 \%)$

$75(78.9 \%)$

$57(60.0 \%)$

$76(80.0 \%)$

$67(70.5 \%$

$34(35.8 \%)$

TABLE 3: Changes to systems and processes

$11.6 \%$ of participants indicated that their practice had been at risk of closure at some point due to the strains of the pandemic, while $8.4 \%$ reported they were unsure of this. $83.2 \%$ felt that they had been given sufficient 


\section{Cureus}

autonomy to tailor their services to fulfil the needs of their own patients, staff and premises.

\section{Support from governing bodies}

Figure 3 demonstrates that NHS England was perceived to be the least useful by study respondents ( $57.9 \%$ scoring $\geqslant 5 / 10)$. Similarly, over half $(56.8 \%)$ of respondents highlighted that they had not received adequate PPE. In contrast, local CCG support was described as the most helpful intervention $(80.0 \%$ scoring $\geqslant 5 / 10)$, whilst the provision of guidelines ranked in the middle $(75.8 \%$ scoring $\geqslant 5 / 10)$.

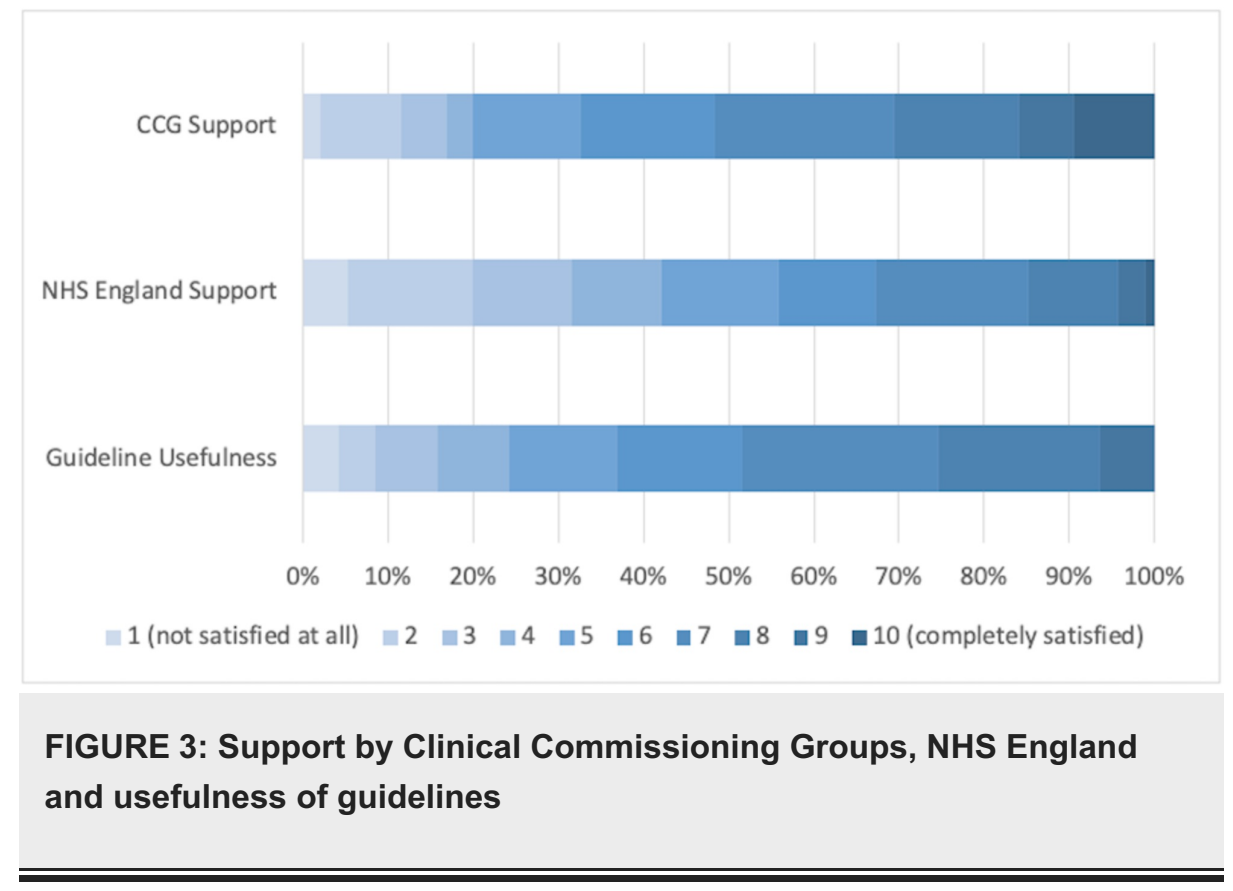

As highlighted in Figure 4, 66.3\% described greater collaboration with their primary care network (PCN) members as a result of COVID-19 (scoring $\geqslant 5 / 10$ ).

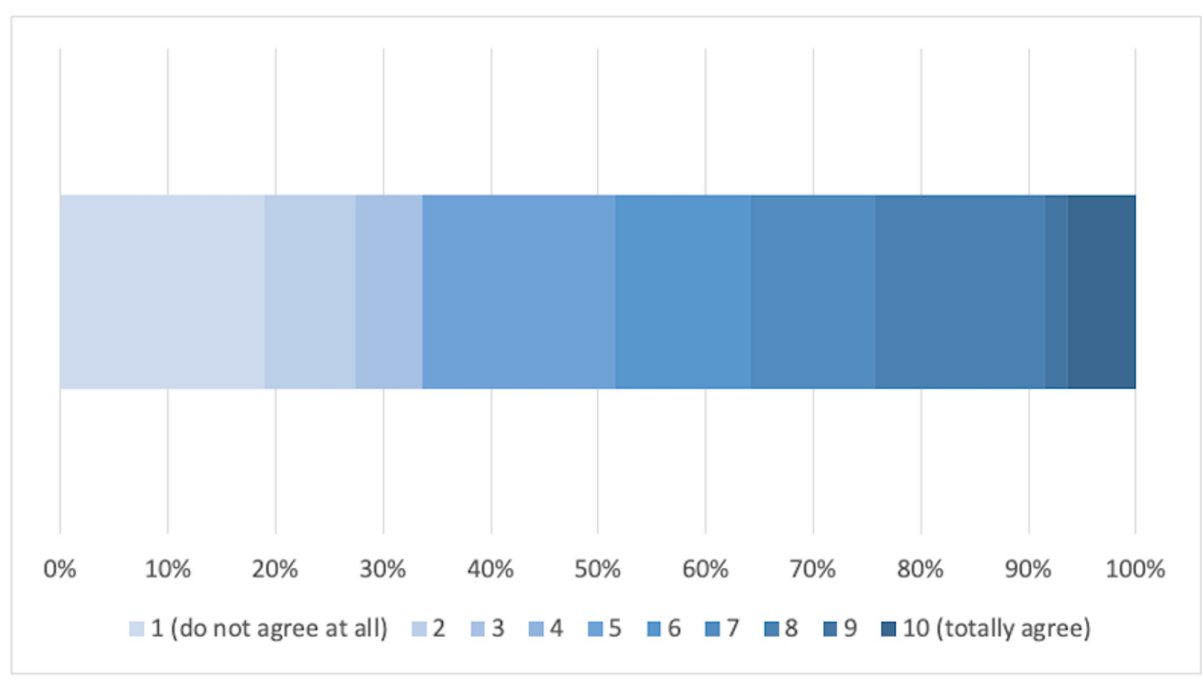

FIGURE 4: Has the response to COVID-19 led to closer integration within a primary care network (PCN) structure?

\section{Proposals to continue beyond the pandemic}

The most common aspirations described by both GPs and PMs were to maintain remote patient consultations (59\%) and remote triage (19\%). There was also a desire for greater working from home (12\%), closer working with the CCG, Local Medical Committee (LMC) and PCN (6\%) but at the same time a request for more autonomy and flexibility (5\%). 
Other reported positive changes include remote patient monitoring, e.g. blood pressure and peak flow (3\%), additional support (3\%) and increased respect from governing bodies (2\%), simplification of death certification, cremation forms and remote viewing of the deceased (3\%), reduced patient expectations (2\%) and reduced abuse of NHS services (4\%), remote team meetings (2\%), fewer tick box exercises such as Care Quality Commission (CQC) visits (2\%), longer consultation times (2\%), streamlined appraisal and revalidation processes ( 1 respondent), increased preparation for winters ( 1 respondent), more regular cleaning (1 respondent) and daily briefings (1 respondent).

\section{Support required to maintain positive changes}

The most cited was a request for increased funding and a simplification to the financial structures supporting primary care (21\%). 20\% felt that significant ongoing patient education is required. Two highlighted examples were education to reduce the medicalisation of social issues and promote self-care as well as teaching patients about how to access the most appropriate resources for their particular issues. $18 \%$ demanded improved IT infrastructure, including computers, webcams and better connectivity, as well as a more responsive technical support (10\%). 11\% called for increased training (especially in IT) and a further $11 \%$ sought greater autonomy with a reduced regulatory burden. 13\% wanted assurance of greater backing from the regulators (NHS England, CQC, and GMC) in the event of complaints arising directly through the rapid and dramatic rise in the use of telemedicine.

\section{Discussion}

This cross-sectional survey of almost 100 GPs and PMs within LLR found that the COVID-19 pandemic has certainly triggered dramatic changes within primary care. Arguably the most significant was the adoption of teleconsultations, in particular video consults, with largely positive feedback from both patients and GPs. Study findings suggests COVID-19 has substantially accelerated the pace of technological change within NHS primary care with the 'right' to digital services by 2024 [6] effectively occurring four years earlier. In addition, practice premises, systems, and processes have also required significant modifications during the pandemic in order to ensure the provision of safe clinical care, e.g. reception screens, one-way patient flow, and greater infection control measures.

However, despite their ability to introduce such widescale change virtually overnight, it was concerning to find that over $10 \%$ of respondents described how the extra strains had placed their practice at risk of closure. Moreover, over half of respondents felt that they were not provided with adequate PPE for their personal safety or that of their staff. Indeed, over a quarter had required absence directly as a result of the pandemic. Perception of the support provided by NHS England and the CCGs was rather mixed, although additional guidelines were broadly welcomed.

The most requested enduring modifications relate to remote working, universal triage, and remote consultations. However, in order to support such largescale permanent change, respondents felt that a different funding and financial structure is required because GPs should not be penalised for providing less face-to-face access. There is also a need for an improved IT infrastructure because currently, the images are often of unacceptable quality. In addition, there was a call for greater patient education, not only in terms of them being able to use remote IT solutions but also in terms of their expectations because some patients remained insistent on face-to-face meetings. Moreover, whilst there is a clear desire to retain large scale teleconsultations beyond the pandemic, significant apprehension was also expressed about their longerterm safety profile. Doctors wanted greater training in telephone and video consultations as well as a detailed evaluation to ensure that important cues and diagnoses were not being overlooked. The art of history taking is complex and takes many years to master and it was felt important not to lose that in this rush to digitalise. Other doctors described how consulting remotely was also less personal and it was more difficult to portray their empathy. Thus, overall, there was a request for a more supportive regulatory environment whist these new modes of consultation become more widely accepted by our patients and better evaluated by our profession.

This study provides a snapshot of the opinions of primary care professionals within one area of the UK. A larger nationwide study may reveal significant regional differences. Although almost 100 survey responses were received, it is disappointing that the actual response rate was only $7 \%$. This was partly expected because of the shear length of the survey. However, it may also be that the clinical pressures of the pandemic and the need to keep abreast of numerous new guidelines as well providing daily situation reports to the CCG were important deterrents. Nevertheless, what was missing in quantity was appropriately compensated for by the quality of the responses received because most participants were highly experienced and had clearly taken considerable time in providing detailed feedback. A further limitation is that the patient perspective was obtained second hand from NHS professionals, but a direct user survey is proposed. Finally, this is a relatively early canvasing of opinions, but the results may be very different if a follow-up review is conducted in 12 months' time.

\section{Conclusions}

Overall, the authors conclude that COVID-19 has placed significant strain upon NHS primary care. However, 


\section{Cureus}

the pandemic has also substantially accelerated the pace of change with a virtually overnight revolution. The long-term fear amongst many GPs and PMs is that there may be insufficient financial and clinical backing from regulatory bodies to support such rapid and far-reaching changes.

\section{Appendices}

\section{Learning from COVID-19}

This confidential 5-10 minute questionnaire aims to identify and consolidate any positive changes that you may have introduced in your surgery in response to the current pandemic. We appreciate your co-operation in completing this form by 14 th June.

*Required

1. Your Job Title *
GP Partner
Salaried GP
$\bigcirc$ Locum GP
$\bigcirc$ Practice Manager

2. Do you consider yourself as belonging to a BAME group? *

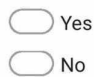

3. Years of NHS experience *

$$
\begin{aligned}
& \square<5 \text { years } \\
& \square 5 \text { - } 10 \text { years } \\
& \square>10 \text { years }
\end{aligned}
$$

4. Have you been working during the COVID-19 pandemic? *

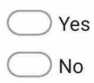

FIGURE 5: Google Forms Survey Page 1 


\section{Cureus}

5. If yes, how much of your work has been done from home?

\begin{tabular}{|c|c|c|c|c|c|c|c|c|c|c|}
\hline 1 & 2 & 3 & 4 & 5 & 6 & 7 & 8 & 9 & 10 & \\
\hline $0 \% \bigcirc$ & $\infty$ & 0 & & & & 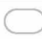 & 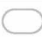 & $\infty$ & D & $100 \%$ \\
\hline
\end{tabular}

6. Have you required any time off work due to COVID-19 symptoms or contact? *

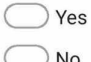

$\bigcirc$ No

7. Did your practice introduce or increase usage of remote patient consultations? *

$\bigcirc$ Yes

$\bigcirc$ No

8. If yes, please estimate the $\%$ increase in remote patient consultations?

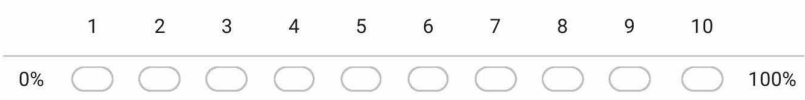

9. Pre-COVID: which modes of consultation were you using? *

$\square$ Face-to-face
$\square$ Video
$\square$ Telephone
$\square$ Email
Other: $\square$

FIGURE 6: Google Forms Survey Page 2 


\section{Cureus}

10. During the pandemic: which modes of consultation have you been using? *

$$
\begin{aligned}
& \square \text { Face-to-face } \\
& \square \text { Video } \\
& \square \text { Telephone } \\
& \square \text { Email } \\
& \text { Other: } \square
\end{aligned}
$$

11. Post-COVID: which modes of consultation do you plan to use? *

$$
\begin{aligned}
& \square \text { Face-to-face } \\
& \square \text { Video } \\
& \square \text { Telephone } \\
& \square \text { Email } \\
& \text { Other: } \square
\end{aligned}
$$

12. Please share any feedback from your clinicians on the use of remote patient consultations *

13. Please share any patient feedback on their remote consultations *

FIGURE 7: Google Forms Survey Page 3 


\section{Cureus}

14. How satisfied were you with the guidance provided on the use of technology to deliver remote healthcare? *

$\begin{array}{llllllllllll}1 & 2 & 3 & 4 & 5 & 6 & 7 & 8 & 9 & 10 & \\ \text { Not satisfied } \square & \square & \square & \square & \square & \square & \square & \square & \square & \square\end{array}$

15. Do you feel you have received adequate PPE? *

$\bigodot_{\text {No }}$

16. What modifications have you required to your premises? *

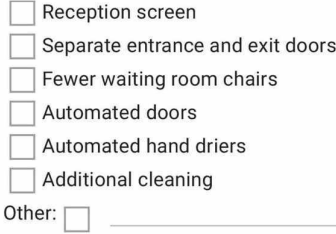




\section{Cureus}

17. How did your systems/processes change due to the pandemic? *

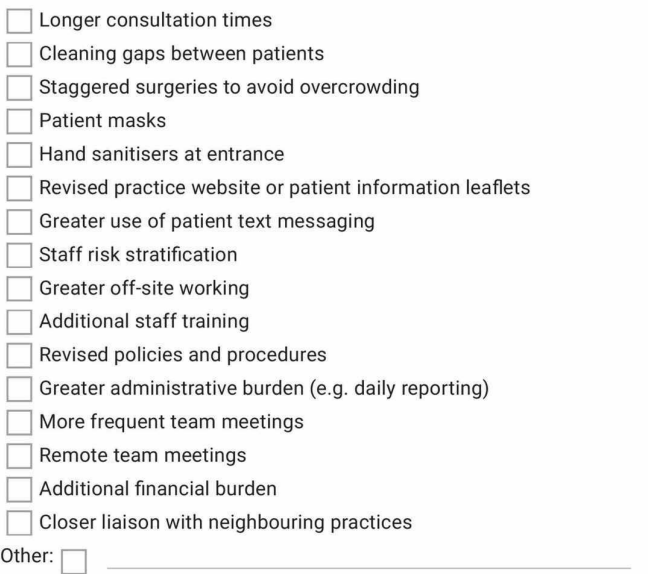

18. Was your surgery ever at risk of closure due to the strain of the pandemic? *

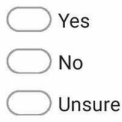

19. Do you work in the City or the County? *

$\bigotimes_{\text {County }}$

FIGURE 9: Google Forms Survey Page 5 


\section{Cureus}

20. Did you feel well supported by your CCG? *

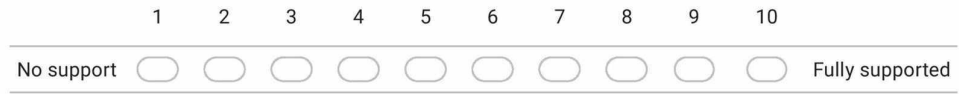

21. Did you feel well supported by NHS England? *

$\begin{array}{llllllllllll}1 & 2 & 3 & 4 & 5 & 6 & 7 & 8 & 9 & 10 \\ \text { No support } \square & \square & \square & \square & \square & \square & \square & \square & \square\end{array}$

22. How useful were the various NHSE/PHE/CCG guidelines? *

$\begin{array}{llllllllllll}1 & 2 & 3 & 4 & 5 & 6 & 7 & 8 & 9 & 10 & \\ \text { Not useful at all } \square & \square & \square & \square & \square & \square & \square & \square & \square & \square & \text { Fully satisfied }\end{array}$

23. Do you feel you were given sufficient autonomy to tailor your services to suit your patients, staffing $\mathrm{c}$ premises? *
$\bigcirc$ Yes
$\bigcirc$ No

24. Has your response to the COVID-19 pandemic allowed for closer integration within a PCN structure?

$\begin{array}{llllllllllll} & 1 & 2 & 3 & 4 & 5 & 6 & 7 & 8 & 9 & 10 & \\ \text { No change at all } \square & \square & \square & \square & \square & \square & \square & \square & \square & \text { Better than before }\end{array}$

FIGURE 10: Google Forms Survey Page 6 


\section{Cureus}

27. Further comments on any changes that have worked well/not been successful, any lessons learnt or please share any case studies? *

Thank you for your feedback

This content is neither created nor endorsed by Google.

FIGURE 11: Google Forms Survey Page 7

\section{Additional Information \\ Disclosures}

Human subjects: Consent was obtained by all participants in this study. Animal subjects: All authors have confirmed that this study did not involve animal subjects or tissue. Conflicts of interest: In compliance with the ICMJE uniform disclosure form, all authors declare the following: Payment/services info: All authors have declared that no financial support was received from any organization for the submitted work. Financial relationships: All authors have declared that they have no financial relationships at present or within the previous three years with any organizations that might have an interest in the submitted work. Other relationships: All authors have declared that there are no other relationships or activities that could appear to have influenced the submitted work.

\section{Acknowledgements}

We would like to thank all the survey respondents. 


\section{Cureus}

\section{References}

1. Shehata M, Zhao S, Gill P: Epidemics and primary care in the UK. Fam Med Community Health. 2020, 8:e000343. 10.1136/fmch-2020-000343

2. Shortell SM, Addicott R, Walsh N, Ham C: The NHS five year forward view: lessons from the United States in developing new care models. BMJ. 2015, 350:h2005. 10.1136/bmj.h2005

3. Pawa J, Robson J, Hull S: Building managed primary care practice networks to deliver better clinical care: a qualitative semi-structured interview study. Br J Gen Pract. 2017, 67:e764-e774. 10.3399/bjgp17X692597

4. Trethewey SP, Beck KJ, Symonds RF: Video consultations in UK primary care in response to the COVID-19 pandemic. Br J Gen Pract. 2020, 70:228-229. 10.3399/bjgp20X709505

5. Razai MS, Doerholt K, Ladhani S, Oakeshott P: Coronavirus disease 2019 (covid-19): a guide for UK GPs . BMJ. 2020, 368:m800. 10.1136/bmj.m800

6. O'Cathail M, Sivanandan MA, Diver C, Patel P, Christian J: The use of patient-facing teleconsultations in the National Health Service: scoping review. JMIR Med Inform. 2020, 8:e15380. 10.2196/15380 